\title{
Crystal structure of caesium tetraphenylborate, $\left(\mathrm{C}_{24} \mathrm{H}_{20} \mathrm{~B}\right) \mathrm{Cs}$
}

\author{
J. C. Bryan
}

Chemical and Analytical Sciences Division, Oak Ridge National Laboratory, Oak Ridge, TN 37831-6119, USA

Received June 6, 2000, CCDC-No. $1267 / 470$

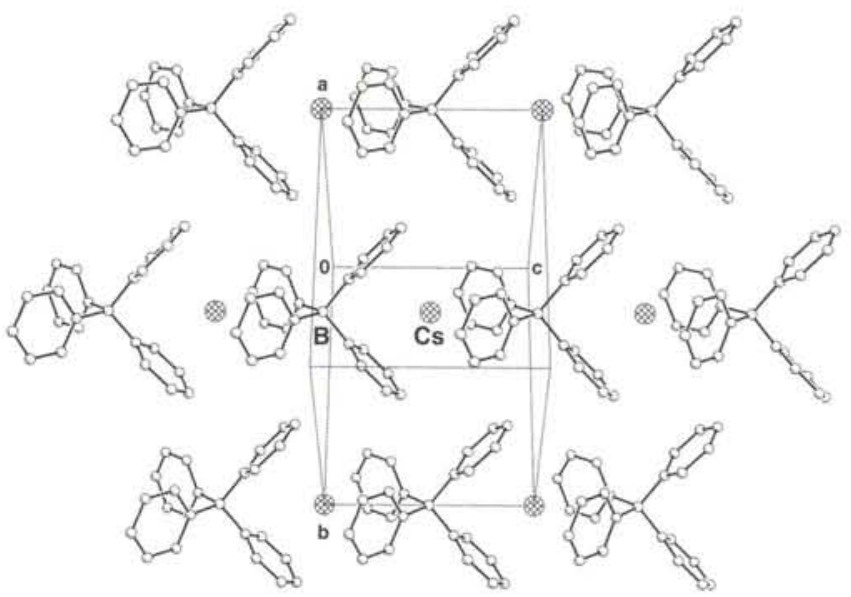

\section{Abstract}

$\mathrm{C}_{24} \mathrm{H}_{20} \mathrm{BCs}$, tetragonal, $\overline{14} 2 \mathrm{~m}$ (No. 121), $a=11.1647(5) \AA$, $c=8.3352(4) \AA, V=1039.0 \AA^{3}, Z=2, R_{\mathrm{gt}}(F)=0.013$, $w R_{\text {ref }}\left(F^{2}\right)=0.033, T=100 \mathrm{~K}$.

\section{Source of material}

The title compound was prepared by mixing aqueous solutions of $\mathrm{CsCl}(0.24 \mathrm{M})$ and $\mathrm{NaBPh}_{4}(0.34 \mathrm{M})$. The resulting precipitate was washed with water and air dried. Crystals were grown from slow evaporation of a acetone/1,2-dichloroethane (2:1) solution.

\section{Discussion}

The separation of radioactive caesium from nuclear wastes, through precipitation of its tetraphenylborate salt, is being considered for large scale application [1]. Despite the present importance of this solid, its crystal structure has not been determined, even though the lattice parameters of this, and several other tetraphenylborate salts, were determined more than 40 years ago [2]. As part of our continuing investigations into separations of large cations and cation- $\pi$ interactions, we decided to determine this structure.

The structure of the title compound bears close resemblance to the previously reported tetraphenylborate salts of rubidium [3] and potassium [4]. All three crystallize in $\bar{I} \overline{4} 2 \mathrm{~m}$ and exhibit the same overall structure. The metal cation rests in one of the clefts between two phenyl rings. Symmetry generates another tetraphenylborate anion which occupies the remainder of the cation's coordination sphere, thus forming a one-dimensional linear chain along the $c$-axis. The cation is therefore coordinated by tetrahedral arrangement of phenyl rings, with the cation-centroid distance decreasing significantly only in the potassium structure (Cs $3.16 \AA$, Rb $3.17 \AA, \mathrm{K} 2.99 \AA$ ). The caesium-centroid distance reported here is among the shortest published [5], and is considerably shorter than the $3.41 \AA$ recently calculated for caesium-benzene [6].

Table 1. Data collection and handling.

\section{Crystal:}

Wavelength:

$\mu$ :

Diffractometer, scan mode: $2 \theta_{\max }$ :

$N(h k l)_{\text {measured }}, N(h k l)_{\text {unique: }}$ Criterion for $I_{\mathrm{obs}}, N(h k l)_{\mathrm{gl}}$ : $N(\text { param })_{\text {refined: }}$

Programs:

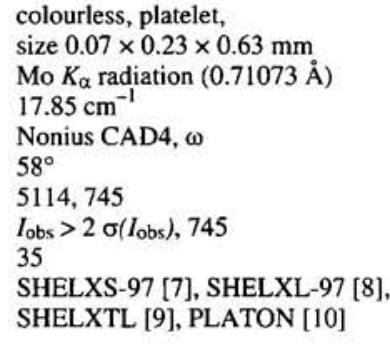

Table 2. Atomic coordinates and displacement parameters (in $\AA^{2}$ ).

\begin{tabular}{llrlll}
\hline Atom & Site & \multicolumn{1}{c}{$x$} & \multicolumn{1}{c}{$y$} & $z$ & $U_{\text {iso }}$ \\
\hline $\mathrm{H}(2)$ & $16 j$ & -0.0319 & -0.2242 & -0.3553 & 0.018 \\
$\mathrm{H}(3)$ & $16 j$ & 0.0715 & -0.328 & -0.1576 & 0.022 \\
$\mathrm{H}(4)$ & $8 i$ & 0.2526 & $-x$ & -0.0557 & 0.025 \\
\hline
\end{tabular}

Table 3. Atomic coordinates and displacement parameters (in $\AA^{2}$ ).

\begin{tabular}{|c|c|c|c|c|c|c|c|c|c|c|}
\hline Atom & Site & $x$ & $y$ & $z$ & $U_{11}$ & $U_{22}$ & $U_{33}$ & $U_{12}$ & $U_{13}$ & $U_{23}$ \\
\hline Cs & $2 a$ & 0 & 0 & 0 & $0.0183(1)$ & $U_{11}$ & $0.0087(1)$ & 0 & 0 & 0 \\
\hline$C(1)$ & $8 i$ & $0.0821(1)$ & $-x$ & $-0.3787(2)$ & $0.0122(4)$ & $U_{11}$ & $0.0105(7)$ & $0.0012(5)$ & $0.0003(4)$ & $-U_{13}$ \\
\hline $\mathrm{C}(2)$ & $16 j$ & $0.0409(1)$ & $-0.1918(1)$ & $-0.3155(2)$ & $0.0172(5)$ & $0.0137(5)$ & $0.0139(5)$ & $0.0000(4)$ & $-0.0003(4)$ & $-0.0007(4)$ \\
\hline$C(3)$ & $16 j$ & $0.1028(1)$ & $-0.2546(1)$ & $-0.1972(2)$ & $0.0237(6)$ & $0.0147(6)$ & $0.0172(6)$ & $0.0020(4)$ & $-0.0005(5)$ & $0.0038(5)$ \\
\hline$C(4)$ & $8 i$ & $0.2103(1)$ & $-x$ & $-0.1367(3)$ & $0.0217(5)$ & $U_{11}$ & $0.0180(9)$ & $0.0066(7)$ & $-0.0037(5)$ & $-U_{13}$ \\
\hline B & $2 b$ & 0 & 0 & $1 / 2$ & $0.0113(7)$ & $U_{11}$ & $0.009(1)$ & 0 & 0 & 0 \\
\hline
\end{tabular}

\footnotetext{
* Correspondence author (e-mail: bryanjc@ornl.gov)
} 
Acknowledgments. This research was sponsored by the Division of Chemical Sciences, Geosciences, and Biosciences, Office of Basic Energy Sciences, U.S. Department of Energy, under contract DE-AC05-00OR22725 with Oak Ridge National Laboratory, managed and operated by UT-Battelle, LLC.

\section{References}

1. Peterson, R. A.; Gaddis, J. L.: Investigation into Optimal Conditions for Cross-Flow Filtration of High-Level Nuclear Waste. Sep. Sci. Technol. 34 (1999) 1507-1520.

2. Arnott, S.; Abrahams, S. C.: The Lattice Constants of the Alkali Salts of Tetraphenyl Boron. Acta Crystallogr. 11 (1958) 449-450.

3. Ozol, Y.; Vimba, S.; Ievins, A.: Crystal Structure of Rubidium Tetraphenylborate. Kristallografiya 7 (1962) 362-365.
4. Hoffmann, K.; Weiss, E.: Über Metall-Alkyl- und Aryl-Verbindungen XVI. Die Kristallstrukturen von Kaliumtetraphenylborat, $\mathrm{K}\left[\mathrm{B}\left(\mathrm{C}_{6} \mathrm{H}_{5}\right)_{4}\right]$ und Tetramethylammonium-tetraphenylborat, $\left[\mathrm{N}\left(\mathrm{CH}_{3}\right)_{4}\right]\left[\mathrm{B}\left(\mathrm{C}_{6} \mathrm{H}_{5}\right)_{4}\right]$. J. Organomet. Chem. 67 (1974) 221-228.

5. Based on a search of the Cambridge Structural Database System V5.19, April 2000 release.

6. Nicholas, J. B.; Hay, B. P.; Dixon, D. A.: Ab Initio Molecular Orbital Study of Cation- $\pi$ Binding between the Alkali-Metal Cations and Benzene. J. Phys. Chem. A. 103 (1999) 1394-1400.

7. Sheldrick, G. M.: SHELXS-97. Program for the solution of crystal structures. Universität Göttingen, Germany 1997.

8. Sheldrick, G. M.: SHELXL-97. Program for Crystal Structure Refinement. Universität Göttingen, Germany 1997.

9. Bruker: SHELXTL 5.10/IRIX. Bruker Analytical X-ray Instruments Inc. Madison, Wisconsin, USA 1997.

10. Spek, A. L.: PLATON. A Multi-Purpose Crystallographic Tool Universiteit Utrecht, The Netherlands 1999. 